\title{
Probleme mit dem Klassenkampf - Marxistische Theorie und soziale Bewegungen
}

Probleme mit dem Klassenkampf in einer Zeitschrift, die sich "Probleme des Klassenkampfs« nennt? Diese Spannung kann zumindest doppelt gedeutet werden. Es kann heißen, daß eine politisch-wissenschaftliche Konzeption, die ursprünglich den Anspruch hatte, durch Analyse der empirischen Prozesse der Konstitution der Arbeiterklasse einen Beitrag zur Beschleunigung des Auflösungsprozesses der bürgerlichen Gesellschaft zu leisten so noch in PROKLA 6 -, daß diese Konzeption anläßlich der relativen Stagnation der traditionellen Arbeiterbewegung und des Aufschwungs der neuen sozialen Bewegungen in die Krise geraten ist und einer Neubestimmung bedarf. Es kann auch heißen, daß diese Konzeption im Kern immer noch für richtig gehalten wird, die Arbeiterklasse der entscheidende Emanzipationsträger ist, die neuen sozialen Bewegungen letztlich Teilelemente in dem Konstitutionsprozeß der Arbeiterklasse darstellen, und deshalb diejenigen, die dies nicht sehen, ihre Probleme mit dem Klassenkampf haben.

Mag sein, daß es noch mehr Nuancen gibt. Der Gegensatz jedoch zwischen einer Emanzipationskonzeption, die Abschied vom Proletariat (A. Gorz) nimmt, und einer Emanzipationskonzeption, die ihr Zuhause beim Proletariat hat, durchzieht die meisten politischen Diskussionen der Neuen Linken, die sich auf dem kleinsten gemeinsamen Nenner doch darin definiert, daß sie im weitesten Sinn sozialistische Praxis auf dem Fundament marxistischer Gesellschaftstheorie und -analyse gründen will. Dieser Gegensatz ist gewiß auch in subtiler Form in den Editorials seit PROKLA 36 aufzuspüren, die im Spannungsfeld zwischen Arbeiterbewegung und neuen sozialen Bewegungen auf die Krise des Marxismus unterschiedliche Antworten zu geben versuchten. Dieser Gegensatz war deutlich sichtbar auf der 1. Sozialistischen Konferenz in Kassel, auf der wohl theoretisch die Vereinbarkeit von Ökologie und Marxismus allenthalben herausgestellt wurde, praktisch jedoch zwischen Ökologie- und Gewerkschaftsbewegung antithetisch Gattungsfrage gegen Klassenfrage stand. Dieser Gegensatz war überdeutlich auf der 2. Sozialistischen Konfernz in Marburg, auf der eine sachliche Kontroverse über die aufgebrochenen Probleme des Klassenkampfs und zwar nicht lediglich aufgrund des Verhaltens der Marxistischen Gruppe - nicht ausgetragen werden konnte, sondern unter dem Schirm der Einheit scheinbar unversöhnliche politische Standpunkte frontal gegenüberstanden. Am wenigsten dürfte die Fluchtbewegung nach vorn in eine undefinierte Funktion innerhalb einer existierenden Friedensbewegung eine Klärung der aufgebrochenen theoretischen und praktischen Probleme herbeiführen. Gerade umgekehrt: Nur in der wohl politisch orientierten, aber von unmittelbarem Handlungsdruck entlasteten Diskussion ist eine solche Klärung denkbar.

In diesem Sinn hatten wir eine pauschale Beantwortung der Krise des Marxismus abgelehnt und stattdessen eine Grundsatzdebatte gefordert, »...in der zunächst einmal alle Dimensionen, die in der Krise des Marxismus aufgebrochen sind, ohne sektiererische Abgrenzungen ernst genommen, präzisiert und diskutiert werden (sollten), in der Hoffnung, daß aus einem solchen Diskurs wirklich neue und richtungsweisende Antworten entstehen.«(PRO- 
KLA 36, Editorial) Im Rahmen einer solchen Grundsatzdebatte muß vor allen Dingen geklärt werden, inwieweit die in der Formulierung »Probleme des Klassenkampfs« enthaltene politisch-wissenschaftliche Konzeption, in Orientierung auf den Konstitutionsprozeß der Arbeiterklasse durch marxistische Analysen den Auflösungsprozeß der bürgerlichen Gesellschaft voranzutreiben, noch trägt, oder inwieweit sie nicht mehr trägt. Die Vorstellung eines derartigen wissenschaftlichen Sozialismus ist ja nicht nur als Leitlinie unserer Zeitschrift untergründig zweifelhaft geworden, sondern in großen Teilen der marxistischen Neuen Linken in der Bundesrepublik, und drückt sich symptomatisch darin aus, wenn unter der Hand aus »Beiträgen zum wissenschaftlichen Sozialismus schlicht »Sozialismus« wird.

In Zweifel geraten ist dabei nicht, daß Analysen der gegenwärtigen gesellschaftlichen Verhältnisse auf der Grundlage der Maxxistischen Theorie - bei allen empirischen, theoretischen und methodischen Schwierigkeiten - prinzipiell zur Aufklärung über die Gesellschaftsbedingungen, unter denen praktisches Handeln stattfindet, unerläßlich sind. In Zweifel geraten ist jedoch, in welchem Sinn und für wen diese marxistischen Analysen emanzipatorisch handlungsorientierend sind, d.h. in welchem Sinn sie eine sozialistische Praxis begründen. Und dieser Zweifel kann nicht von der Hand gewiesen werden, weil die pauschalisierende Orientierung auf »die Arbeiterklasse angesichts der realen sozialen und politischen Prozesse brüchig geworden ist, und weil der Marxismus in Form einer an Universitäten und Forschungsinstituten isoliert und spezialistisch durchgeführten Gesellschaftsanalyse die ihm zugewiesene Funktion einer sozialistischen Handlungsorientierung in sozialen Bewegungen kaum wahrnehmen kann. Der Marxismus zerfällt in institutionalisierten Akademismus und politische Pragmatik. Darin zerfällt aber auch der Anspruch des Marxismus, praktische Theorie einer emanzipatorischen Gesellschaftsveränderung zu sein.

Mit diesen Bemerkungen ist der Problemhorizont umrissen, in dem die Zusammenstellung der Beiträge in diesem Heft unter dem Thema: »Marxistische Theorie und soziale Bewegungen« vorgenommen worden ist. Zunächst wirft $J$. Hoffmann unter dem Stichwort der skorporatistischen Blockbildung « die politische Frage auf, inwieweit nicht auf dem Hintergrund der ökonomischen Entwicklung der Bundesrepublik und ihrer Folgen für die soziale Struktur der Arbeiterklasse der DGB in Richtung einer Gewerkschaftspolitik geht, die unter dem Druck der verschärften Weltmarktkonkurrenz zunehmend die Konkurrenz zwischen verschiedenen Fraktionen der Arbeiterklasse ausdrückt, statt sich am Ziel einer einheitlichen Interessenvertretung aller Arbeiter unter Einschluß von A rbeitslosen und Marginalisierten zu orientieren. Die damit aritkulierte Voraussetzung, daß eine sozialistische Politik sich nicht jenseits der traditionellen Arbeiterbewegung bewegen kann, wird in Form der Gorz-Kritik durch F.O. Wolf als allgemeines theoretisches Problem formuliert: wie nämlich unter Annahme der Gorzschen radikalen Absage an jede geschichtsphilosophische Überhöhung des Proletariats im Marxismus der 2. und 3. Internationale ein Ausweg aus der damit zugespitzten Krise des Marxismus im Festhalten an der Marxschen Intention der Aufhebung der kapitalistischen Form der Vergesellschaftung konzipierbar ist, ohne in die umgekehrte geschichtsphilosophische Überhöhung der neuen sozialen Bewegungen bei Gorz zu geraten. A. Evers und Z. Szankay thematisieren anschließend eine weitere zentrale Dimension der Krise des Marxismus: das aktuelle Auseinanderfallen von marxistischer Theorie und dem in sozialen Bewegungen inkorporierten sozialen Wissen wird als Folge einer spezifischen Marxismustradition - wie auch in PROKLA vorherrschend - gesehen, die mit der Vorstellung der Superiorität marxistischen Wissens soziales W issen ignoriert und 
zugleich, wie subtil auch immer, die Führung gegenüber sozialen Bewegungen beansprucht.

Um die Krise des Marxismus nicht nur aus westdeutscher Sicht, sondern auch in ihrer englischen Variante zu sehen, gibt WW. Spobn einen kritischen Überblick über die politische, marxismustheoretische und geschich tswissenschaftliche Kontroverse zwischen E.P. Thompson und P. Anderson: Ersterer wird inzwischen durch seine sozialhistorischen Analysen, seine Althusser-Polemik und sein Engagement gegen die aktuelle atomare Aufrüstung auch hierzulande zu einem Begriff; und letzterer als langjähriger Herausgeber der "New Left Review ist durch seine Marxismusinterpretation und seine Studien zum Feudalismus und zum absolutistischen Staat auch kein Unbekannter mehr - doch ihre Kontroverse um das politische Selbstverständnis der Neuen Linken wird kaum wahrgenommen. Wiederum unter dem Gesichtspunkt des Verhältnisses von marxistischer Theorie und sozialer Bewegung will dann M. Vester, der seit vielen Jahren das Thompsonsche Werk in Deutschland publik zu machen suchte, in seinem Beitrag zum Verhältnis von Engels zur deutschen Arbeiterbewegung auch historisch zeigen, in welch problematischer Weise die Engelssche Konzeption des wissenschaftlichen Sozialismus Einfluß auf die deutsche Arbeiterklasse und ihre hierarchischen Organisationsstrukturen hatte. R. Paris setzt anschließend in der Kritik an $\mathrm{O}$. Rammstedts soziologischer Theorie sozialer Bewegungen die historische Thematik Thompsons und Yesters der politischen und kulturellen 'Infrastruktur' der Arbeiterklasse soziologisch fort, indem er als konstitutives Merkmal sozialer Bewegungen im Unterschied zu Gefolgschaftśsparteien ihre basisdemokratische Öffentlichkeitsstruktur herausarbeitet. Schließlich macht $H$. Heise in ihrem Diskussionsbeitrag zu dem vieldiskutierten Aufsatz von A. Mohl in PROKLA 36, der eine emanzipationstheoretische Lesart des Marxschen 'Kapital' vorschlug, wiederum eine wissenschaftlich-sozialistische Lesart geltend. Auch die abschließ3end dokumentierten Texte von $E$. Balibar zur Position der KPF gegenüber den Arbeitsimmigranten, der prompt zu seinem Ausschluß aus der Partei führte, und von K.S. Karol zur Tragödie der Althussers stehen im direkten Zusammenhang zur Krise des Marxismus: Sie sollen ein Gegengewicht gegen die im Zeichen des Schicksals von Poulantzas und Althusser sich häufende - etwa durch die Thompsonsche Althusser-Polemik ungewollt beförderte - Denunziation marxistischer Theorie überhaupt schaffen.

Auf die Krise des Marxismus gibt es keine fertigen Antworten, und gerade sie wären gegenwärtig suspekt. Deshalb haben wir versucht, aus verschiedenen marxistischen Traditionen Beiträge zu den relevanten Dimensionen der Krise des Marxismus zusammenzustellen. Sie mögen Aufforderung sein, eine produktive Auseinandersetzung um das politische Selbstverständnis der marxistischen Linken zu führen.

Die Redaktion 